\title{
A MRAC Principle for a Single-Link Electrically Driven Robot with Parameter Uncertainties
}

\author{
Carlos Aguilar-Avelar and Javier Moreno-Valenzuela \\ Instituto Politécnico Nacional-CITEDI, Ave. Instituto Politécnico Nacional 1310, Nueva Tijuana, 22435 Tijuana, BC, Mexico
}

Correspondence should be addressed to Javier Moreno-Valenzuela; moreno@citedi.mx

Received 2 July 2016; Accepted 14 November 2016; Published 15 January 2017

Academic Editor: Sigurdur F. Hafstein

Copyright (C) 2017 C. Aguilar-Avelar and J. Moreno-Valenzuela. This is an open access article distributed under the Creative Commons Attribution License, which permits unrestricted use, distribution, and reproduction in any medium, provided the original work is properly cited.

\begin{abstract}
In this paper, a model reference adaptive control (MRAC) principle for a one-degree-of-freedom rigid-link electrically driven robot is presented. The proposed control methodology addresses the problem of trajectory tracking with parameter uncertainties in the dynamic model of the system and proposes adaptation laws for the electrical and mechanical parameters. Closed-loop stability is rigorously discussed, proving that the tracking error trajectories converge to the origin exponentially. With the aim of performing experimental comparisons, two control schemes are also revisited theoretically and experimentally: one is an algorithm previously reported in the literature and the other is an adaptive controller derived under the assumption that the electrical dynamics of the actuator are negligible. All the discussed controllers have been implemented in an experimental setup consisting in a rigid-link robot actuated with brushed DC motor. The comparison indicates that better results are obtained with the new MRAC scheme.
\end{abstract}

\section{Introduction}

Adaptive control is a design technique focused on highperformance applications in control of dynamical systems, in order to deal with parametric uncertainties characterized by a set of unknown constant parameters. On the other hand, the design of adaptive controllers requires knowing the structure of the dynamic model of the system [1].

An advantage of adaptive control, considering that the parameters of the plant changes during operation, is that the controller compensates the changes in the model parameters achieving better performance. In a nonadaptive system, where the parameters of the plant are considered constants, these changes can compromise the good performance of the overall system. Another advantage of the adaptive control systems is that the knowledge of the plant parameters to design the controller is not necessary [2].

Direct current (DC) motors are widely used as actuators in different kind of robots. Due to its dynamics, DC motors can be described appropriately by a linear dynamic model, which is sensitive to parametric variations, noise interference, and parametric uncertainties. Therefore, conventional control schemes can not yield a convenient performance in the presence of the aforementioned disturbances [3]. Taking into account these facts, this document presents a new adaptive control scheme in addition to a theoretical and experimental comparative study of adaptive algorithms for the trajectorytracking control of a one-degree-of-freedom (DOF) rigidlink electrically driven (RLED) robot.

In [4], an adaptive tracking controller for a DC motor was presented. Simulation results are given, where the DC motor shaft follows a desired sinusoidal trajectory with an acceptable tracking performance. In [5], a summary of previous works on trajectory-tracking control for manipulator robots was presented, in which the electrical dynamics of the actuators are neglected in the design procedure. In [6], the idea of considering the electrical dynamics of the actuators as a part of the system dynamics was introduced, and a robust controller for the trajectory tracking was presented. This controller guarantees that the tracking error is uniformly ultimately bounded. In addition, an adaptive controller for a rigid robot was presented in [5], where the electrical dynamics of the actuators are considered in the controller design. In [7], the electrical dynamics were added to the controller design for an adaptive algorithm previously reported in literature. This is for the position regulation problem on rigid robots 
actuated with a brushed DC motor. However, only simulation results were provided. In [8], a formal stability analysis was introduced for a voltage based controller designed for manipulator robots, although the problem of parametric uncertainties was not addressed.

Let us notice that much of the recent literature in the control of DC motors does not take into account the load dynamics but pays attention to other important problems with practical relevance. In $[9,10]$, the velocity control of a DC motor was addressed by taking into account the dynamics of a DC-DC power converter. Those approaches were supported by real-time experiments. The integration of barrier Lyapunov function to avoid the violation of state constraints was used in [11] for the development of DC motor controller. In [12], an output-feedback control was proposed for the positioning problem of a lineal actuator driven by a DC motor, where the saturation of the control input and the presence of nonlinear friction are considered in the development of the new methodology.

The application of model reference adaptive control (MRAC) for a RLED robot is introduced in this document. In general, the MRAC-based adaptive controllers are designed using a reference model which describes the desired characteristics of the plant to be controlled. The use of a reference model facilitates the analysis of the adaptive system and provides a stability framework, since the controller design and stability analysis was performed in two steps. Regarding this, the MRAC scheme was analyzed in [13], in the presence of unmodelled dynamics, where the analysis showed the existence of robustness and this fact was confirmed by means of simulations. In [14], the application of a MRAC scheme for an induction motor was presented, shown to be robust to parametric variations. In [15], a MRAC-based controller was presented in order to solve the speed control problem of a DC motor. In this case, the classic adaptation scheme is replaced with fuzzy linear adaptation to improve the system performance at low speed and variable load conditions. Nevertheless, results are only validated with simulations. In [16], an adaptive output-feedback controller that belongs to the class of MRAC schemes was presented. This algorithm is applied to manipulators neglecting the actuator dynamics and uses a linear observer to estimate the states for a manipulator. In [17], a MRAC scheme was presented in order to control robot manipulators. However, the electrical dynamics of the actuators are not considered in the controller design. More recently, the velocity control of DC motor was explored in [18] by using a MRAC scheme together with a variable structure extension. However, electrical dynamics were also neglected. Other interesting applications of the MRAC philosophy for nonlinear systems can be found in, for example, [19-22].

More recently, intelligent control techniques have been applied to control this kind of systems. For instance, fuzzy and neural networks based controllers were introduced in [23-32]. However, in most cases, only simulation results are provided. In [33-35], some sliding-mode based controllers were presented for robot manipulators, where the electrical dynamics of the actuator are neglected. However, in [36], a neural-network-based terminal sliding-mode control scheme was proposed for robotic manipulators including actuator dynamics. In [37], a robust optimal voltage control of electrically driven robot manipulators was presented. Particle swarm optimization is used to optimize the control design parameters. Also, a comparative study between voltage and torque based controllers is presented, which confirms the superiority of the voltage control strategy. In [38], a robust discrete repetitive control of electrically driven robot manipulators was introduced, where the control problem is the tracking of a periodic trajectory. However, in all of the aforementioned work, only simulation results were given, making comparing with the controllers presented in this document difficult.

The main contributions of this document are the inclusion of the electrical dynamics of the actuator in the control law and the problem of parametric uncertainties, which are considered in a united form, in addition to the design and analysis of a new adaptive control algorithm for the trajectory-tracking control of a 1-DOF RLED robot. Specifically, the novel adaptive algorithm presented is based on a MRAC principle. It is noteworthy that the application of the MRAC scheme for the control of RLED robots deserves a deeper study since only a few studies have been reported.

Additionally, an experimental performance comparison of our solution with respect to some other adaptive algorithms is presented. For the comparative study, this paper revisits two adaptive controllers: an algorithm previously reported in literature [39] and an adaptive controller derived under the assumption that the electrical dynamics of the actuator is negligible. The new MRAC scheme and the others are tested in real time in an experimental platform consisting in a 1-DOF RLED robot which is affected by gravitational force.

This paper is organized as follows: Section 2 is devoted to summarizing the RLED robot dynamic model. In addition, a brief discussion about the control goal is presented. The proposed MRAC scheme is described in Section 3. An adaptive controller previously reported in literature is given in Section 4. In Section 5, an adaptive controller obtained from the assumption that the electrical dynamics of the actuator are negligible is presented. A brief description of the experimental platform, results of the real-time implementation of the new controller, and the performance comparison are presented in Section 6. Finally, in Section 7, some concluding remarks are given.

\section{RLED Robot Dynamic Model and Control Goal}

The dynamic model of a 1-DOF RLED robot actuated by a brushed DC motor is presented as in [39] and is written as

$$
\begin{aligned}
J \ddot{q}+B \dot{q}+N \sin (q) & =i, \\
L \frac{d}{d t} i+R i+K_{B} \dot{q} & =v,
\end{aligned}
$$

where $J$ is the grouped rotor inertia, $B$ is the grouped viscous friction, $N$ is the constant of grouped gravitational 
load, $L$ is the inductance, $R$ is the electric resistance, and $K_{B}$ is the back electromotive force constant, all these being positive constants. Furthermore, $q(t), \dot{q}(t)$, and $\ddot{q}(t)$ represent position, velocity, and acceleration, respectively. Finally, $i(t)$ and $v(t)$ represent current and the input voltage, respectively.

It is possible to represent (1)-(2) as a linear regression model, which separates the constant parameters from the states. This representation is especially useful in parametric identification and in the design of adaptive controllers. Then, the mechanical part of the system model described in (1) is rewritten in a linear regression form as

$$
Y_{m}(q, \dot{q}, \ddot{q}) \boldsymbol{\theta}_{m}=i
$$

where

$$
\begin{aligned}
& Y_{m}=\left[\begin{array}{lll}
\ddot{q} & \dot{q} & \sin (q)
\end{array}\right], \\
& \boldsymbol{\theta}_{m}=\left[\begin{array}{lll}
J & B & N
\end{array}\right]^{T},
\end{aligned}
$$

with $Y_{m}$ in (4) being the regression matrix of the mechanical part and $\boldsymbol{\theta}_{m}$ in (5) being the vector of constant parameters of the mechanical part.

Similarly, the electrical part of the system model described in (2) can be represented as

$$
Y_{e}\left(\frac{d}{d t} i, i, \dot{q}\right) \boldsymbol{\theta}_{e}=v,
$$

where

$$
\begin{aligned}
& Y_{e}=\left[\begin{array}{lll}
\frac{d}{d t} i & i & \dot{q}
\end{array}\right], \\
& \boldsymbol{\theta}_{e}=\left[\begin{array}{lll}
L & R & K_{B}
\end{array}\right]^{T},
\end{aligned}
$$

with $Y_{e}$ in (7) being the regression matrix of the electrical part and $\boldsymbol{\theta}_{e}$ in (8) being the vector of constant parameters of the electrical part.

Let us consider a time-varying twice differentiable signal $q_{d}(t)$, which denotes the desired trajectory of the joint position. Then, the control goal consists in ensuring that the trajectory-tracking error $\tilde{q}(t)=q_{d}(t)-q(t)$ satisfies

$$
\lim _{t \rightarrow \infty} \tilde{q}(t)=0
$$

This paper introduces three controllers that ensure limit (9).

\section{Proposed MRAC Principle}

A new MRAC-based algorithm is presented in this section, where, in the first stage of design, a control action is proposed for the tracking between the real model and the reference model. After this, a complementary control action is defined for the tracking between the reference model and a desired trajectory. Hence, the control objective defined in (9) is satisfied.
3.1. Reference Model. The proposed reference model for systems (1)-(2) is defined as

$$
\begin{aligned}
\widehat{J} \ddot{q}_{r}+\widehat{B} \dot{q}_{r}+\widehat{N} \sin (q) & =i_{r}, \\
\widehat{L} \frac{d}{d t} i_{r}+\widehat{R} i_{r}+\widehat{K}_{B} \dot{q}_{r} & =v+u,
\end{aligned}
$$

where $\widehat{J}(t)$ is the estimated grouped rotor inertia, $\widehat{B}(t)$ is the estimated grouped viscous friction, $\widehat{N}(t)$ is the estimated constant of grouped gravitational load, $\widehat{L}(t)$ is the estimated inductance, $\widehat{R}(t)$ is the estimated electric resistance, and $\widehat{K}_{B}(t)$ is the estimated back electromotive force constant, all these being positive constants. Furthermore, $\dot{q}_{r}(t)$ and $\ddot{q}_{r}(t)$ represent velocity and acceleration of the reference model, respectively. Also, $i_{r}(t)$ and $v(t)$ represent current and the input voltage of the reference model, respectively. Finally, $u(t)$ is an auxiliary control signal to be defined. Note that $v(t)$ represents also the input of systems (1)-(2).

It is possible to express the reference dynamic model described in (10)-(11) as a linear regression model as well. Then, the mechanical part of the reference model in (10) is rewritten in a linear regression form as

$$
Y_{m r}\left(q, \dot{q}_{r}, \ddot{q}_{r}\right) \widehat{\boldsymbol{\theta}}_{m r}=i_{r}
$$

where

$$
\begin{aligned}
& Y_{m r}=\left[\begin{array}{lll}
\ddot{q}_{r} & \dot{q}_{r} & \sin (q)
\end{array}\right], \\
& \widehat{\boldsymbol{\theta}}_{m r}=\left[\begin{array}{lll}
\widehat{J} & \widehat{B} & \widehat{N}
\end{array}\right]^{T},
\end{aligned}
$$

with $Y_{m r}(t)$ being the regression matrix of the mechanical part of the reference model and $\widehat{\boldsymbol{\theta}}_{m r}(t)$ being the vector of estimated parameters of the mechanical part of the reference model.

Similarly, the electrical part of the reference model described in (11) can be expressed as

$$
Y_{e r}\left(\frac{d}{d t} i_{r}, i_{r}, \dot{q}_{r}\right) \hat{\boldsymbol{\theta}}_{e r}=v+u,
$$

where

$$
\begin{aligned}
& Y_{e r}=\left[\begin{array}{lll}
\frac{d}{d t} i_{r} & i_{r} & \dot{q}_{r}
\end{array}\right], \\
& \widehat{\boldsymbol{\theta}}_{e r}=\left[\begin{array}{lll}
\widehat{L} & \widehat{R} & \widehat{K}_{B}
\end{array}\right]^{T},
\end{aligned}
$$

with $Y_{e r}(t)$ being the regression matrix of the electrical part of the reference model and $\widehat{\boldsymbol{\theta}}_{e r}(t)$ being the vector of estimated parameters of the electrical part of the reference model.

3.2. Error Model. Now we proceed to compute the open-loop error dynamics of the electrical and mechanical part. The error model of the mechanical part is given by the difference between the mechanical part of the reference model in (12) and the mechanical part of the real model in (3). Analogously, the error model of the electrical part is given by the difference 
between the electrical part of the reference model in (15) and the electrical part of the real model in (6).

The error model is expressed as

$$
\begin{aligned}
Y_{m r}\left(q, \dot{q}_{r}, \ddot{q}_{r}\right) \widehat{\boldsymbol{\theta}}_{m r}-Y_{m}(q, \dot{q}, \ddot{q}) \boldsymbol{\theta}_{m} & =\bar{i}, \\
Y_{e r}\left(\frac{d}{d t} i_{r}, i_{r}, \dot{q}_{r}\right) \widehat{\boldsymbol{\theta}}_{e r}-Y_{e}\left(\frac{d i}{d t}, i, \dot{q}\right) \boldsymbol{\theta}_{e} & =u,
\end{aligned}
$$

where $\bar{i}=i_{r}-i$.

The adaptation errors are defined as follows:

$$
\begin{gathered}
\widetilde{\boldsymbol{\theta}}_{m r}=\boldsymbol{\theta}_{m}-\widehat{\boldsymbol{\theta}}_{m r} \in \mathbb{R}^{3}, \\
\tilde{\boldsymbol{\theta}}_{e r}=\boldsymbol{\theta}_{e}-\widehat{\boldsymbol{\theta}}_{e r} \in \mathbb{R}^{3} .
\end{gathered}
$$

In order to define the error model in terms of the parameter adaptation error, it is possible to substitute the expression of $\widehat{\boldsymbol{\theta}}_{m r}(t)$ from (19) into the error model in (17), such that

$$
\begin{gathered}
Y_{m r}\left(q, \dot{q}_{r}, \ddot{q}_{r}\right) \boldsymbol{\theta}_{m}-Y_{m}(q, \dot{q}, \ddot{q}) \boldsymbol{\theta}_{m} \\
-Y_{m r}\left(q, \dot{q}_{r}, \ddot{q}_{r}\right) \tilde{\boldsymbol{\theta}}_{m r}=\bar{i} .
\end{gathered}
$$

Writing explicitly the first two terms in (21), doing suitable cancellations, and defining the position error between the reference model and the real model as

$$
\bar{q}=q_{r}-q
$$

the error model of the mechanical part can be written as

$$
J \ddot{\bar{q}}+B \dot{\bar{q}}-Y_{m r}\left(q, \dot{q}_{r}, \ddot{q}_{r}\right) \tilde{\boldsymbol{\theta}}_{m r}=\bar{i} .
$$

It is worth remarking that if the parameter estimation error of the mechanical part $\widetilde{\boldsymbol{\theta}}_{m r}(t)$ is equal to zero in (23), then the remaining elements describe the error model for a reference model with known parameters.

Similarly proceeding, the error model of the electrical part in (18) can be rewritten as

$$
L \frac{d}{d t} \bar{i}+R \bar{i}+K_{B} \dot{\bar{q}}-Y_{e r}\left(\frac{d}{d t} i_{r}, i_{r}, \dot{q}_{r}\right) \widetilde{\boldsymbol{\theta}}_{e r}=u .
$$

As well as for the mechanical part of the error model, notice that if the parameter estimation error of the electrical part $\widetilde{\boldsymbol{\theta}}_{e r}(t)$ is equal to zero in (24), then the remaining elements in (24) describe the error model for a reference model with known parameters. Systems (23)-(24) describe the tracking error dynamics between the reference model (10)-(11) and the actual model (1)-(2).

3.3. Controller for the Tracking between Reference Model and Real Model. As can be seen from the error dynamics (23)-(24), the input $u(t)$ must be designed such that the convergence of the electric current error $\bar{i}(t)$ and the position error $\bar{q}(t)$ is ensured. In other words, the control signal $u(t)$ is introduced in order to ensure the tracking between the reference model (10)-(11) and the actual model (1)-(2). With this aim, $u(t)$ is proposed as feed-forward compensation in terms of the electrical dynamics plus a proportional-integral part in terms of a change of variable $\rho(t)$; that is,

$$
u=\widehat{L} \dot{y}+\widehat{R} y-k_{0} \rho-k_{i} \xi,
$$

where $k_{0}$ and $k_{i}$ are positive constants and the change of variable,

$$
\rho=\bar{i}-y,
$$

is defined in order to ensure that the current error $\bar{i}(t)$ introduces the compensation $y(t)$ to the mechanical error dynamics (23). In other words, the proportional part $k_{0} \rho$ and the integral part $k_{i} \xi$, with

$$
\dot{\xi}=\rho
$$

are introduced to ensure that $\bar{i}=y$. On the other hand, the compensation $y(t)$ given as

$$
y=-k_{d} z-k_{p} \bar{q}
$$

introduces a proportional term in $\bar{q}(t)$ and a filtered derivative compensation $z(t)$ defined as

$$
\dot{z}=-k_{f}(z-\dot{\bar{q}})
$$

with $k_{p}, k_{d}$, and $k_{f}$ being positive constants.

Taking into account the definition of the electrical adaptation error $\widetilde{\boldsymbol{\theta}}_{e r}(t)$ in (20), in particular, the definitions of $\widetilde{L}(t)$ and $\widetilde{R}(t)$, the control signal $u(t)$ in (25) can be expressed as

$$
u=L \dot{y}+R y-k_{0} \rho-k_{i} \xi-\widetilde{L} \dot{y}-\widetilde{R} y .
$$

Substituting the definitions of $\bar{i}(t)$ in (26) and $y(t)$ in (28) into (23), the expression of the mechanical part of the error model is rewritten as

$$
J \ddot{\bar{q}}+B \dot{\bar{q}}+k_{d} z+k_{p} \bar{q}-Y_{m r} \tilde{\boldsymbol{\theta}}_{m r}=\rho .
$$

Analogously, by substituting the control signal $u(t)$ in (30) into the the electrical part of the error model in (24), this yields

$$
L \dot{\rho}+\left[R+k_{0}\right] \rho+K_{B} \dot{\bar{q}}+k_{i} \xi-Y_{e u} \tilde{\boldsymbol{\theta}}_{e r}=0
$$

where

$$
Y_{e u}=\left[\frac{d}{d t}\left(i_{r}-y\right)\left(i_{r}-y\right) \dot{q}_{r}\right] .
$$

The closed-loop systems (31)-(32) result from applying the controller $u(t)$ in (25) to the open-loop system in (23)-(24).

3.4. Adaptation Law. We propose the following adaptation laws

$$
\begin{aligned}
\frac{d}{d t} \tilde{\boldsymbol{\theta}}_{m r} & =-\Gamma_{m r} Y_{m r}^{T}(\dot{\bar{q}}+\alpha \bar{q}), \\
\frac{d}{d t} \widetilde{\boldsymbol{\theta}}_{e r} & =-\Gamma_{e r} Y_{e u}^{T} \rho,
\end{aligned}
$$


where $\alpha$ is a positive constant sufficiently small, $Y_{m r}(t)$ is defined in (13), $Y_{e u}(t)$ is defined in (33), and

$$
\begin{gathered}
\Gamma_{m r}=\operatorname{diag}\left(\gamma_{m r_{1}}, \gamma_{m r_{2}}, \gamma_{m r_{3}}\right)>0, \\
\Gamma_{e r}=\operatorname{diag}\left(\gamma_{e r_{1}}, \gamma_{e r_{2}}, \gamma_{e r_{3}}\right)>0
\end{gathered}
$$

are defined as the adaptation gains of the mechanical and electrical part, respectively.

3.5. Analysis for Tracking between the Reference Model and Real Model. We propose the following energy function:

$$
\begin{aligned}
\mathscr{V}= & \frac{\varepsilon}{2} L \rho^{2}+\frac{\varepsilon}{2} k_{i} \xi^{2}+\frac{1}{2} J \dot{\bar{q}}^{2}+\frac{1}{2} k_{p} \bar{q}^{2}+\frac{1}{2} k_{f}^{-1} k_{d} z^{2} \\
& +\alpha J \dot{\bar{q}}+\frac{\alpha}{2} B \bar{q}^{2}+\frac{1}{2} \widetilde{\boldsymbol{\theta}}_{m r}^{T} \Gamma_{m r}^{-1} \widetilde{\boldsymbol{\theta}}_{m r}+\frac{\varepsilon}{2} \widetilde{\boldsymbol{\theta}}_{e r}^{T} \Gamma_{e r}^{-1} \widetilde{\boldsymbol{\theta}}_{e r}
\end{aligned}
$$

with $\bar{q}(t)$ defined in (22), $\rho(t)$ in (26), $\xi(t)$ in (27), $z(t)$ in (29), $\widetilde{\boldsymbol{\theta}}_{m r}(t)$ in (19), and $\widetilde{\boldsymbol{\theta}}_{e r}(t)$ in (20).

It is possible to prove that, for $\alpha$ small enough, $\mathscr{V}$ in (37) is a positive definite and radially unbounded function in terms of the states of the reference model error described by (27), (29), (31), and (32), as well as the adaptation laws in (34)-(35).

To ensure that the tracking error between the reference model and the actual model, which is expressed by $\bar{q}(t)$, is a bounded signal, it is required that $\mathscr{V}$ in (37) be a decreasing function, that is, the time derivative $\dot{\mathscr{V}}$ to be negative semidefinite.

By performing all the suitable substitutions and conducting the algebra, the time derivative of the function (37) is given by

$$
\begin{aligned}
\dot{\mathscr{V}}= & -\varepsilon\left[R+k_{0}\right] \rho^{2}-\varepsilon K_{B} \rho \dot{\bar{q}}-B \dot{\bar{q}}^{2}+\dot{\bar{q}} \rho-k_{d} z^{2} \\
& +\alpha \dot{\bar{q}}^{2}-\alpha k_{d} \bar{q} z-\alpha k_{p} \bar{q}^{2}+\alpha \bar{q} \rho,
\end{aligned}
$$

which can be expressed in a matrix form as

$$
\dot{\mathscr{V}}=-\mathbf{x}^{T} \mathbf{P} \mathbf{x}
$$

where

$$
\begin{aligned}
& \mathbf{x}=\left[\begin{array}{cccc}
\bar{q} & \dot{\bar{q}} & z & \rho
\end{array}\right]^{T}, \\
& \mathbf{P}=\left[\begin{array}{cccc}
\alpha k_{p} & 0 & \frac{\alpha k_{d}}{2} & -\frac{\alpha}{2} \\
0 & B-\alpha J & 0 & \frac{\varepsilon K_{B}-1}{2} \\
\frac{\alpha k_{d}}{2} & 0 & k_{d} & 0 \\
-\frac{\alpha}{2} & \frac{\varepsilon K_{B}-1}{2} & 0 & \varepsilon\left[R+k_{0}\right]
\end{array}\right] .
\end{aligned}
$$

Since (39) is a quadratic form, by proving that $\mathbf{P}=\mathbf{P}^{T}$ in (41) is a positive definite matrix, we ensure that $\dot{\mathscr{V}}$ is a negative semidefinite function. We can prove this fact by using Sylvester's criterion to determine conditions to ensure that $\mathbf{P}$ is positive definite. Then, it can be shown that, by selecting sufficiently large values for $k_{p}$ and $k_{0}$, as well as sufficiently small values for $\alpha$ and $\varepsilon$, there exist conditions for $\mathbf{P}$ to be a positive definite matrix. Finally, it is possible to invoke Barbalat's lemma [40] to prove that

$$
\lim _{t \rightarrow \infty} \mathbf{x}(t)=0,
$$

where $\mathbf{x}(t)$ is defined in (40). Hence, the tracking error $\bar{q}(t)$ between the reference model and the real model tends to zero as time $t$ increases.

3.6. Controller for Desired Trajectory Tracking. Once the tracking error between the reference model and the actual model $\bar{q}(t)$ is proven to be convergent, we propose the control voltage $v(t)$ in order to ensure that the reference model position $q_{r}(t)$ tracks a desired time-varying trajectory $q_{d}(t)$. With this aim, the tracking error signals are defined as

$$
\begin{aligned}
& \delta=i_{d}-i_{r}, \\
& e=q_{d}-q_{r},
\end{aligned}
$$

and the control voltage $v(t)$ is proposed as

$$
v=-u+\varphi,
$$

where $u(t)$ is defined in (25) and

$$
\begin{aligned}
\varphi & =\widehat{K}_{B} \dot{q}_{d}+\widehat{L} \frac{d}{d t} i_{d}+\widehat{R} i_{d}+k_{o v} \delta \\
i_{d} & =\widehat{J} \ddot{q}_{d}+\widehat{B} \dot{q}_{d}+\widehat{N} \sin (q)+k_{d v} \dot{e}+k_{p v} e .
\end{aligned}
$$

Proceeding to obtain the closed-loop equations in terms of $\delta(t)$ in (43) and $e(t)$ in (44), we substitute (47) into (43), where, solving for $i_{r}(t)$, we obtain

$$
i_{r}=\widehat{J} \ddot{q}_{d}+\widehat{B} \dot{q}_{d}+\widehat{N} \sin (q)+k_{d v} \dot{e}+k_{p v} e-\delta,
$$

where $k_{p v}$ and $k_{d v}$ are positive constants. Solving (10) for $\ddot{q}_{r}(t)$ and substituting into the second time derivative of the tracking error in (44), one obtains

$$
\ddot{e}=\ddot{q}_{d}-\widehat{J}^{-1}\left[i_{r}-\widehat{B} \dot{q}_{r}-\widehat{N} \sin (q)\right] .
$$

Substituting (48) into (49) and after some simplifications, we obtain the tracking error dynamics of the mechanical part as follows:

$$
\widehat{J} \ddot{e}+\left[\widehat{B}+k_{d v}\right] \dot{e}+k_{p v} e=\delta .
$$

Similarly, to obtain the closed-loop equation for the electrical part in terms of the tracking error $\delta(t)$, we calculate the time derivative of (43), and multiplying by $\widehat{L}(t)$, this yields

$$
\widehat{L} \frac{d}{d t} \delta=\widehat{L}\left[\frac{d}{d t} i_{d}-\frac{d}{d t} i_{r}\right] .
$$

Solving (11) for $(d / d t) i_{r}(t)$, substituting into (51), and performing the algebra, we obtain the error dynamics of the electrical part as follows:

$$
\widehat{L} \dot{\delta}+\left[\widehat{R}+k_{o v}\right] \delta=-\widehat{K}_{B} \dot{e} .
$$


The system formed by (50) and (52) is linear and timevarying. It can be demonstrated that if $\widehat{J}(t), \widehat{B}(t), \widehat{L}(t), \widehat{R}(t)$, and $\widehat{K}_{B}(t)$ are strictly positives for all $t \geq 0$, we have that

$$
\lim _{t \rightarrow \infty}\left[\begin{array}{l}
e(t) \\
\delta(t)
\end{array}\right]=0
$$

with exponential rate of convergence. It is noteworthy that the limits (42) and (53) imply that the control goal in (9) is satisfied.

Finally, it is worth remarking that the total control voltage $v(t)$ to be applied to the open-loop system dynamics in (1)-(2) is given in (45), with the auxiliary control signals $u(t)$ in (25) which ensures the tracking between reference model and real model, $\varphi(t)$ in (46) which introduces the desired dynamics to the system, the parameter adaptation laws $\dot{\tilde{\boldsymbol{\theta}}}_{m r}(t)$ in (34), $\dot{\tilde{\boldsymbol{\theta}}}_{e r}(t)$ in (35), and definitions therein.

\section{Adaptive Controller Proposed by Dawson, Hu, and Burg (1998)}

In this section, an adaptive controller proposed in [39] is presented and the stability analysis is briefly reviewed. The purpose of presenting this algorithm is to make a comparison with respect to the MRAC scheme introduced in this paper. Thus, the trajectory-tracking adaptive controller is written as

$$
v=W_{e} \widehat{\boldsymbol{\theta}}_{e}+K_{e} \tilde{i}+r,
$$

where $K_{e}$ is a positive constant, $r(t)$ is the filtered tracking error given by

$$
r=\dot{\tilde{q}}+\alpha \tilde{q},
$$

with $\alpha$ being a positive constant, $\tilde{q}(t)$ stands for the trajectorytracking error defined as

$$
\tilde{q}=q_{d}-q,
$$

where $q_{d}(t)$ is the desired trajectory, $\widetilde{i}(t)$ is the current error defined by

$$
\widetilde{i}=i_{d}-i,
$$

where $i_{d}(t)$ is the desired current, which is expressed as

$$
i_{d}=W_{\tau} \widehat{\boldsymbol{\theta}}_{\tau}+K_{s} r,
$$

with the positive constant $K_{s}$,

$$
W_{\tau}=\left[\ddot{q}_{d}+\alpha \dot{\tilde{q}} \dot{q} \sin (q)\right]
$$

defined as the regressor of the mechanical part, and

$$
\widehat{\boldsymbol{\theta}}_{\tau}=\left[\begin{array}{lll}
\widehat{J} & \widehat{B} & \widehat{N}
\end{array}\right]^{T} \in \mathbb{R}^{3}
$$

is an estimation of the parameters of the mechanical part $\boldsymbol{\theta}_{\tau}$. Similarly,

$$
\widehat{\boldsymbol{\theta}}_{e}=\left[\begin{array}{llllll}
\frac{\widehat{L}}{J} & \frac{\widehat{L B}}{J} & \widehat{R} & \widehat{K}_{B} & \frac{\widehat{L N}}{J} & \widehat{L}
\end{array}\right]^{T} \in \mathbb{R}^{6}
$$

represents the estimation of $\boldsymbol{\theta}_{e}$, which is a vector of grouped parameters, containing parameters from both mechanical and electrical part. Likewise,

$$
W_{e}=\left[\begin{array}{llllll}
w_{e 1} & w_{e 2} & w_{e 3} & w_{e 4} & w_{e 5} & w_{e 6}
\end{array}\right]
$$

with

$$
\begin{aligned}
w_{e 1}= & \widehat{B} i-K_{s} i-\alpha \widehat{J} i, \\
w_{e 2}= & K_{s} \dot{q}-\widehat{B} \dot{q}+\alpha \widehat{J} \dot{q}, \\
w_{e 3}= & i, \\
w_{e 4}= & \dot{q}, \\
w_{e 5}= & K_{s} \sin (q)-\widehat{B} \sin (q)+\alpha \widehat{J} \sin (q), \\
w_{e 6}= & \widehat{J} \ddot{q}_{d}+\alpha \widehat{J} \ddot{q}_{d}+W_{\tau} \Gamma_{\tau} W_{\tau}^{T} r+K_{s} \ddot{q}_{d}+K_{s} \alpha \dot{\tilde{q}} \\
& +\widehat{N} \dot{q} \cos (q),
\end{aligned}
$$

being the regressor of the vector of grouped parameters.

4.1. Adaptation Law. By defining the adaptation errors as

$$
\begin{aligned}
& \widetilde{\boldsymbol{\theta}}_{\tau}=\boldsymbol{\theta}_{\tau}-\widehat{\boldsymbol{\theta}}_{\tau} \in \mathbb{R}^{3}, \\
& \widetilde{\boldsymbol{\theta}}_{e}=\boldsymbol{\theta}_{e}-\widehat{\boldsymbol{\theta}}_{e} \in \mathbb{R}^{6},
\end{aligned}
$$

the adaptation laws for the update of the mechanical parameters $\widehat{\boldsymbol{\theta}}_{\tau}(t)$ and grouped electrical parameters $\widehat{\boldsymbol{\theta}}_{e}(t)$ are given by

$$
\begin{aligned}
& \frac{d}{d t} \widetilde{\boldsymbol{\theta}}_{\tau}=-\Gamma_{\tau} W_{\tau}^{T} r, \\
& \frac{d}{d t} \widetilde{\boldsymbol{\theta}}_{e}=-\Gamma_{e} W_{e}^{T} \widetilde{i},
\end{aligned}
$$

where

$$
\begin{aligned}
& \Gamma_{\tau}=\operatorname{diag}\left(\gamma_{\tau 1}, \gamma_{\tau 2}, \gamma_{\tau 3}\right)>0, \\
& \Gamma_{e}=\operatorname{diag}\left(\gamma_{e 1}, \gamma_{e 2}, \ldots, \gamma_{e 6}\right)>0
\end{aligned}
$$

are the adaptation gains of the mechanical and electrical parts, respectively.

The closed-loop system is obtained by substituting the control action $v(t)$ defined in (54) into the dynamic model (1)-(2). By expressing in terms of the filtered tracking error $r(t)$, the current error $\widetilde{i}(t)$, and the adaptation errors $\widetilde{\boldsymbol{\theta}}_{\tau}(t)$ and $\tilde{\boldsymbol{\theta}}_{e}(t)$, the closed-loop system is given by

$$
\frac{d}{d t}\left[\begin{array}{c}
r \\
\widetilde{i} \\
\tilde{\boldsymbol{\theta}}_{\tau} \\
\tilde{\boldsymbol{\theta}}_{e}
\end{array}\right]=\left[\begin{array}{c}
J^{-1}\left[W_{\tau} \tilde{\boldsymbol{\theta}}_{\tau}-K_{s} r+\widetilde{i}\right] \\
L^{-1}\left[W_{e} \widetilde{\boldsymbol{\theta}}_{e}-K_{e} \tilde{i}-r\right] \\
-\Gamma_{\tau} W_{\tau}^{T} r \\
-\Gamma_{e} W_{e}^{T} \widetilde{i}^{T}
\end{array}\right] .
$$


4.2. Stability Analysis. To investigate stability of the equilibrium point at the origin of system (67), the following Lyapunov candidate function is proposed:

$$
\mathscr{V}=\frac{1}{2} J r^{2}+\frac{1}{2} L \widetilde{i}^{2}+\frac{1}{2} \tilde{\boldsymbol{\theta}}_{\tau}^{T} \Gamma_{\tau}^{-1} \widetilde{\boldsymbol{\theta}}_{\tau}+\frac{1}{2} \tilde{\boldsymbol{\theta}}_{e}^{T} \Gamma_{e}^{-1} \widetilde{\boldsymbol{\theta}}_{e}
$$

where the time derivative of $\mathscr{V}$ along the trajectories of the closed-loop system (67) is given by

$$
\dot{\mathscr{V}}=-K_{s} r^{2}-K_{e} \tilde{i}^{2}
$$

Hence, $\dot{\mathscr{V}}$ in (69) is negative or zero. Then, the origin of the state space is Lyapunov stable. Invoking Barbalat's lemma is possible to prove that

$$
\lim _{t \rightarrow \infty}\left[\begin{array}{l}
r(t) \\
\tilde{i}(t)
\end{array}\right]=\left[\begin{array}{l}
0 \\
0
\end{array}\right]
$$

is satisfied. Also it can be shown that the tracking error $\widetilde{q}(t)$ approaches zero as time $t$ increases; that is

$$
\lim _{t \rightarrow \infty} \tilde{q}(t)=0
$$

See [39] for further details about the stability analysis.

\section{Adaptive PD Controller Neglecting Electrical Dynamics}

In this section, an adaptive PD controller is presented. This algorithm is derived under the assumption that the electrical dynamics of the actuator are neglected. In literature, this is based on the assumption that the inductance $L$ is sufficiently small, such that the current $i(t)$ can be approached by

$$
i=\frac{v}{R}-\frac{K_{B}}{R} \dot{q}
$$

Substituting (72) into the mechanical part of model (1), the expression

$$
R J \ddot{q}+\left[R B+K_{B}\right] \dot{q}+R N \sin (q)=v
$$

is obtained. Note that system (73) can be controlled by using a PD-type scheme plus adaptive compensation to ensure convergence of the error $\tilde{q}(t)$.

By assuming that the current error $\widetilde{i}(t)$ converges quickly to zero, the resistance $R$ is compensated by the controller, and redefining the parameters of model (73) as

$$
\begin{aligned}
J_{R} & =R J, \\
B_{R} & =R B+K_{B}, \\
N_{R} & =R N,
\end{aligned}
$$

our adaptive controller for system (73) is written as follows:

$$
v=Y_{m d} \widehat{\boldsymbol{\theta}}_{m}+K_{p m} \widetilde{q}+K_{d m} \dot{\tilde{q}}
$$

with

$$
\begin{aligned}
Y_{m d} & =\left[\begin{array}{lll}
\ddot{q}_{d} & \dot{q}_{d} & \sin (q)
\end{array}\right], \\
\widehat{\boldsymbol{\theta}}_{m} & =\left[\begin{array}{lll}
\widehat{J}_{R} & \widehat{B}_{R} & \widehat{N}_{R}
\end{array}\right]^{T},
\end{aligned}
$$

and $\widetilde{q}(t)$ is obtained from (56) and $K_{p m}$ and $K_{d m}$ are defined as positive constants. Note that the controller (75) has a PDtype structure in the position error $\widetilde{q}(t)$.

5.1. Adaptation Law. The adaptation law for the update of the mechanical parameters $\widehat{\boldsymbol{\theta}}_{m}(t)$ is given by

$$
\frac{d}{d t} \widehat{\boldsymbol{\theta}}_{m}=\Gamma_{m} Y_{m d}^{T}[\epsilon \tilde{q}+\dot{\tilde{q}}]
$$

where

$$
\Gamma_{m}=\operatorname{diag}\left(\gamma_{m 1}, \gamma_{m 2}, \gamma_{m 3}\right)>0
$$

is the adaptation gains of the mechanical part and $\epsilon$ is a strictly positive constant.

5.2. Stability Analysis. By defining the adaptation error of the mechanical part as

$$
\widetilde{\boldsymbol{\theta}}_{m}=\boldsymbol{\theta}_{m}-\widehat{\boldsymbol{\theta}}_{m} \in \mathbb{R}^{3},
$$

the closed-loop system formed by the substitution of the controller in (75) into the open-loop system in (73) can be written as

$$
\begin{aligned}
\frac{d}{d t} \widetilde{q} & =\dot{\tilde{q}}, \\
J_{R} \frac{d}{d t} \dot{\tilde{q}} & =-\left[B_{R}+K_{d m}\right] \dot{\tilde{q}}-K_{p m} \widetilde{q}+Y_{m d} \widetilde{\boldsymbol{\theta}}_{m}, \\
\frac{d}{d t} \widetilde{\boldsymbol{\theta}}_{m} & =-\Gamma_{m} Y_{m d}^{T}[\epsilon \tilde{q}+\dot{\tilde{q}}] .
\end{aligned}
$$

It is possible to show that the origin of state space is an equilibrium point.

Let us consider that the constant $\epsilon$ involved in the adaptation law (77) satisfies

$$
\epsilon<\min \left\{\sqrt{\frac{K_{p m}}{J_{R}}}, \frac{K_{p m}\left[B_{R}+K_{d m}\right]}{K_{p m} J_{R}+(1 / 4)\left[B_{R}+K_{d m}\right]^{2}}\right\} .
$$

Furthermore, under (81),

$$
\mathscr{V}=\frac{1}{2} K_{p m} \tilde{q}^{2}+\frac{1}{2} J_{R} \dot{\tilde{q}}^{2}+\epsilon J_{R} \tilde{q} \dot{\tilde{q}}+\frac{1}{2} \tilde{\boldsymbol{\theta}}_{m}^{T} \Gamma_{m}^{-1} \widetilde{\boldsymbol{\theta}}_{m}
$$

is a candidate Lyapunov function for the state space origin of system (80). The time derivative of $\mathscr{V}$ along the closed-loop system trajectories is given in a matrix form by

$$
\dot{\mathscr{V}}=-\left[\begin{array}{c}
\widetilde{q} \\
\dot{\tilde{q}}
\end{array}\right]^{T}
$$

$$
\underbrace{\left[\begin{array}{cc}
\epsilon K_{p m} & -\frac{\epsilon}{2}\left[B_{R}+K_{d m}\right] \\
-\frac{\epsilon}{2}\left[B_{R}+K_{d m}\right] & {\left[B_{R}+K_{d m}\right]-\epsilon J_{R}}
\end{array}\right]}_{Q}\left[\begin{array}{c}
\tilde{q} \\
\dot{\tilde{q}}
\end{array}\right] .
$$


It is possible to show that (81) is a sufficient condition that guarantees the positive definiteness of the matrix $Q$ in (83) and, invoking Barbalat's lemma, it is possible to prove that

$$
\lim _{t \rightarrow \infty}[|\widetilde{q}(t)||\dot{\tilde{q}}(t)|]^{T}=0
$$

is satisfied.

\section{Experimental Evaluation}

In this section, a brief description of the experimental setup and the results obtained from the implementation of the described adaptive controllers are presented.

6.1. Experimental Platform. For the experimental validation of the adaptive controllers presented in this paper, the testbed was constructed using a brushed DC motor from Advanced Motion Controls model MBR2303NI (Nema 34 brushed direct current motor), mounted on an aluminum plate and a pendular load attached to the shaft. See Figure 1 for a picture of the experimental platform. This experimental setup was instrumented with the following components:

(i) A PC with Windows XP

(ii) A servoamplifier Advanced Motion Controls model $30 A 20 A C V$,

(iii) An optical encoder US Digital model HB6M-2500$250-I E-D-H$ with a resolution of 2500 pulses per revolution

(iv) An inductive filter Advanced Motion Controls model BFC10010

(v) A current sensor F.W. Bell model NT-5

(vi) A DAQ Sensoray 626.

The control algorithms were implemented in Simulink and the PC interacts with the DAQ through the Real-Time Windows Target libraries at 1 millisecond of sample time. Finally, Figure 1 shows a diagram of the overall experimental platform.

The desired trajectory was selected as

$$
q_{d}(t)=2 \sin (3 t)+1.5[\mathrm{rad}] .
$$

The physical meaning of this trajectory is a displacement from the downward position to the upward position of the joint, passing through the point where the load is more affected by the gravitational force.

6.2. Control Gains. The selected control gains and adaptation gains are shown in Tables $1-3$. Specifically, Table 1 shows the gains for the proposed MRAC algorithm in (45), Table 2 shows the gains for the adaptive controller in (54) previously introduced by [39], and Table 3 contains the gains for the adaptive PD controller in (75).

The tuning process was carried out in an experimental form, starting with sufficiently small values. Then, each gain was increased looking for an improvement of the trajectorytracking performance. Related to the control gains, small
TABLE 1: Control and adaptation gains for the MRAC algorithm (45).

\begin{tabular}{lc}
\hline Gain & Value \\
\hline$k_{p}$ & 3.0 \\
$k_{i}$ & 1.0 \\
$k_{d}$ & 1.0 \\
$k_{f}$ & 100 \\
$k_{0}$ & 1.0 \\
$k_{p v}$ & 3.0 \\
$k_{o v}$ & 1.0 \\
$k_{d v}$ & 0.3 \\
$\alpha$ & 0.1 \\
$\gamma_{m r 1}$ & 0.01 \\
$\gamma_{m r 2}$ & 0.01 \\
$\gamma_{m r 3}$ & 5.0 \\
$\gamma_{e r 1}$ & 0.01 \\
$\gamma_{e r 2}$ & 0.75 \\
$\gamma_{e r 3}$ & 0.05 \\
\hline
\end{tabular}

TABLE 2: Control and adaptation gains for the DHB algorithm (54).

\begin{tabular}{lc}
\hline Gain & Value \\
\hline$K_{s}$ & 1.0 \\
$K_{e}$ & 1.0 \\
$\alpha$ & 1.0 \\
$\gamma_{\tau 1}$ & 0.01 \\
$\gamma_{\tau 2}$ & 0.01 \\
$\gamma_{\tau 3}$ & 5.0 \\
$\gamma_{e 1}$ & 0.03 \\
$\gamma_{e 2}$ & 0.03 \\
$\gamma_{e 3}$ & 0.3 \\
$\gamma_{e 4}$ & 0.03 \\
$\gamma_{e 5}$ & 0.03 \\
$\gamma_{e 6}$ & 0.03 \\
\hline
\end{tabular}

TABLE 3: Control and adaptation gains for the APD algorithm (75).

\begin{tabular}{lc}
\hline Gain & Value \\
\hline$K_{p m}$ & 3.0 \\
$K_{d m}$ & 1.0 \\
$\epsilon$ & 1.0 \\
$\gamma_{m 1}$ & 0.01 \\
$\gamma_{m 2}$ & 0.01 \\
$\gamma_{m 3}$ & 1.0 \\
\hline
\end{tabular}

values were preferred; this is in order to exhibit the adaptation performance of each algorithm. In general, it is possible to say that all the controllers were tuned in a similar manner.

6.3. Experimental Results. The following shows the obtained experimental results. Figures 2-3 depict the comparison between the desired, the reference, and the actual signals for the position and electric current, respectively, obtained from the application of the proposed model reference adaptive controller in (45). As can be seen, the obtained experimental 


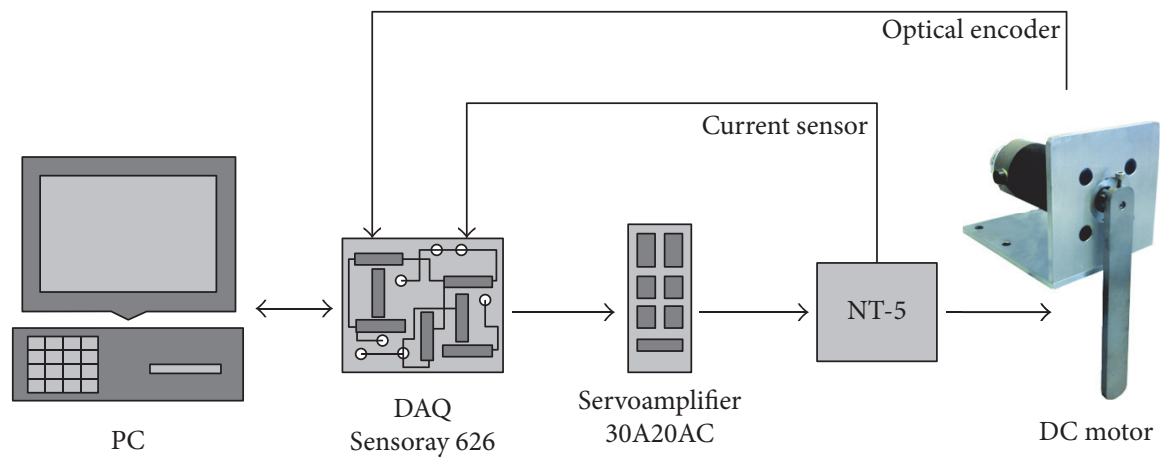

FIGURE 1: Block diagram of the experimental platform of a 1-DOF RLED robot actuated by a brushed DC motor.

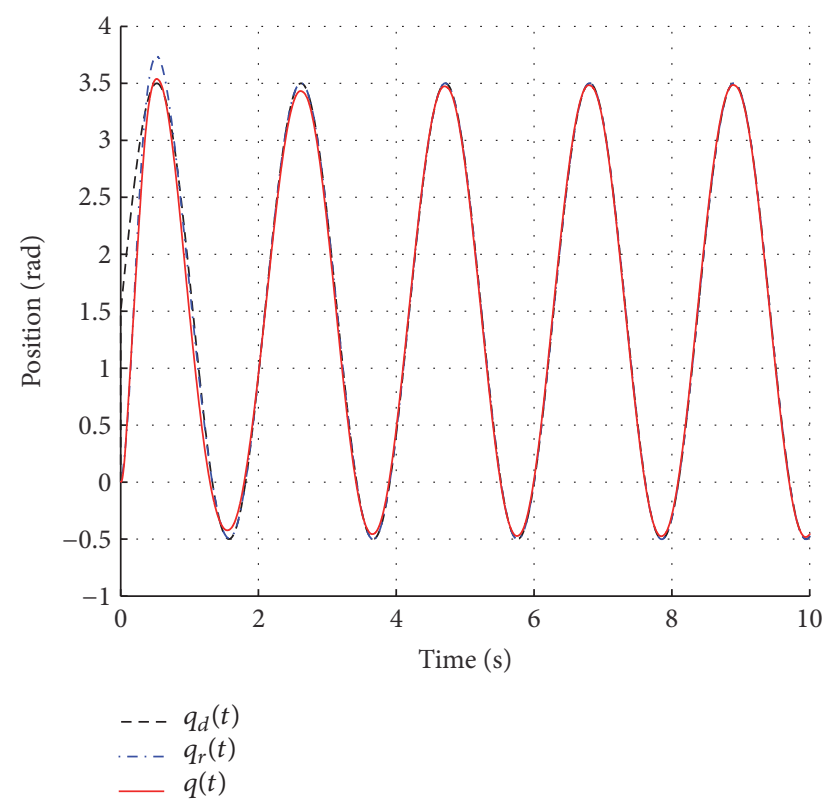

FIGURE 2: Time evolution of the joint position $q(t)$ and the model reference position $q_{r}(t)$ during the tracking of the desired trajectory $q_{d}(t)$ for the MRAC scheme.

results validate the theory, since the tracking between the reference and the desired signals is achieved while the tracking between the actual and the reference signals is also accomplished.

Additionally, Figures 4-6 show the performance comparison of the proposed model reference adaptive controller (MRAC) in (45), the adaptive controller proposed by Dawson, $\mathrm{Hu}$, and Burg (DHB) in (54), and the adaptive PD (APD) controller neglecting the electrical dynamics in (75). Specifically, Figure 4 depicts the comparison between the actual position $q(t)$ and the desired position $q_{d}(t)$ in (85) for the aforementioned adaptive controllers. In Figure 5, the comparison of the electric current signals $i(t)$ can be seen. Finally, Figure 6 depicts the applied control voltage $v(t)$ generated for each control algorithm.

It can be observed from Figure 4 that the MRAC algorithm converges much faster than the others. This can be

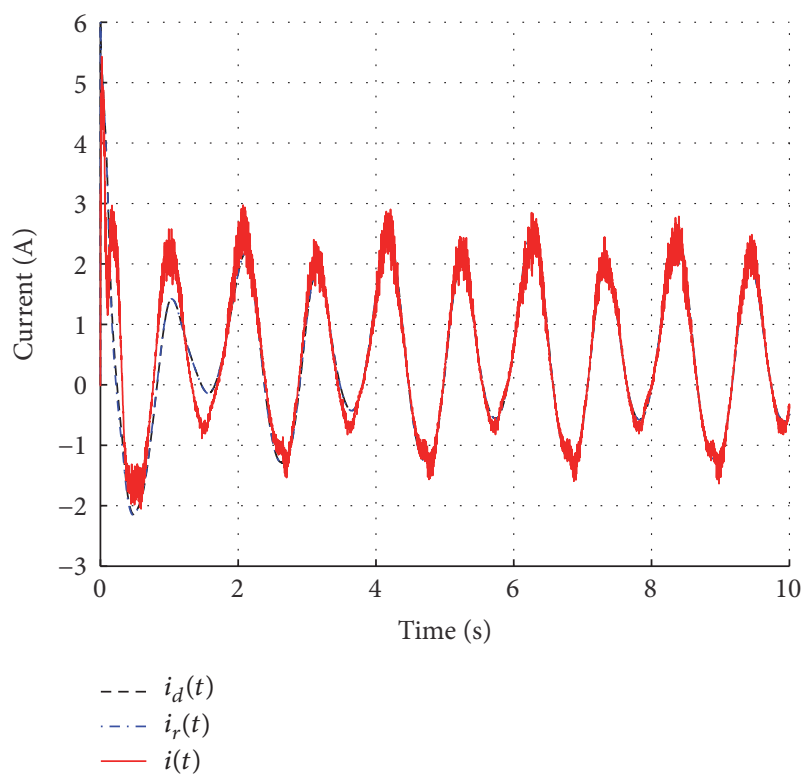

Figure 3: Time evolution of the electric current $i(t)$, the model reference current $i_{r}(t)$, and the desired current $i_{d}(t)$ for the MRAC scheme.

possibly attributed to the overparameterization of the $\mathrm{DHB}$ algorithm and to the simplicity of the APD controller, since the DHB adapts nine parameters against only six parameters adapted by the MRAC, making the MRAC adaptation laws easier to tune. On the other hand, the fact that the APD neglects the dynamics of the electric actuator produces higher error values. It was also observed in Figure 4 that the APD algorithm presents better tracking performance than the DHB controller during the first seconds of experiment, which may be due to the tuning of the DHB controller and the fact that the APD only responds to the magnitude of the position error, without the delay introduced by the electric dynamics of the actuator. However, after the first seconds of experiment, the tracking performance of the DHB becomes much better than the APD.

Furthermore, in Figure 7, the time evolution of the estimated parameters $\widehat{J}(t), \widehat{B}(t)$, and $\widehat{N}(t)$ of the mechanical part is shown for the MRAC and the DHB algorithms. 


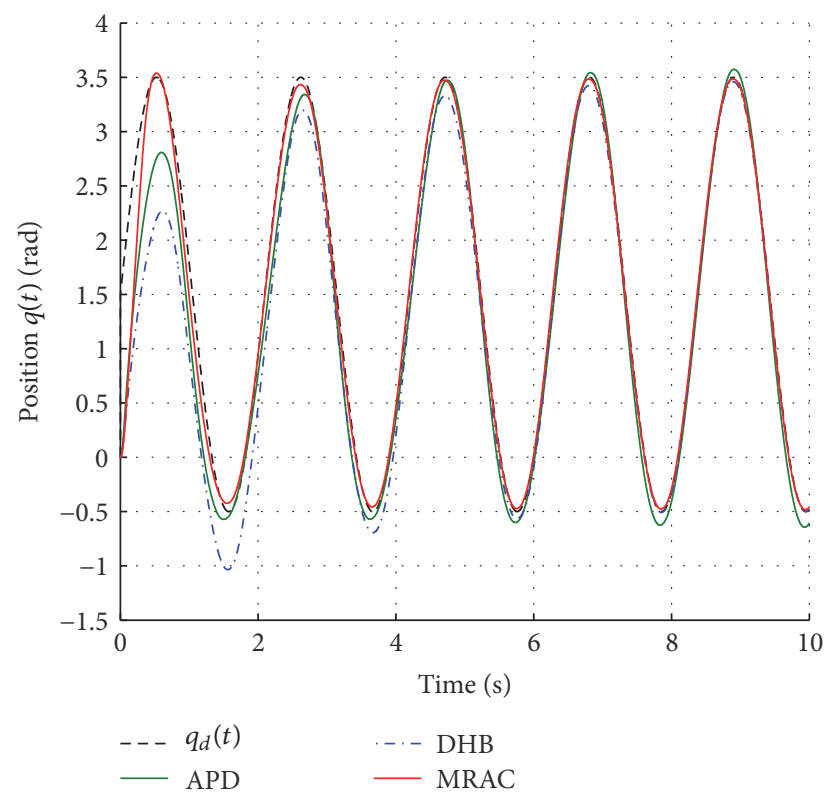

FIGURE 4: Time evolution of the joint position $q(t)$ during the tracking of the desired trajectory $q_{d}(t)$ for all the compared algorithms.

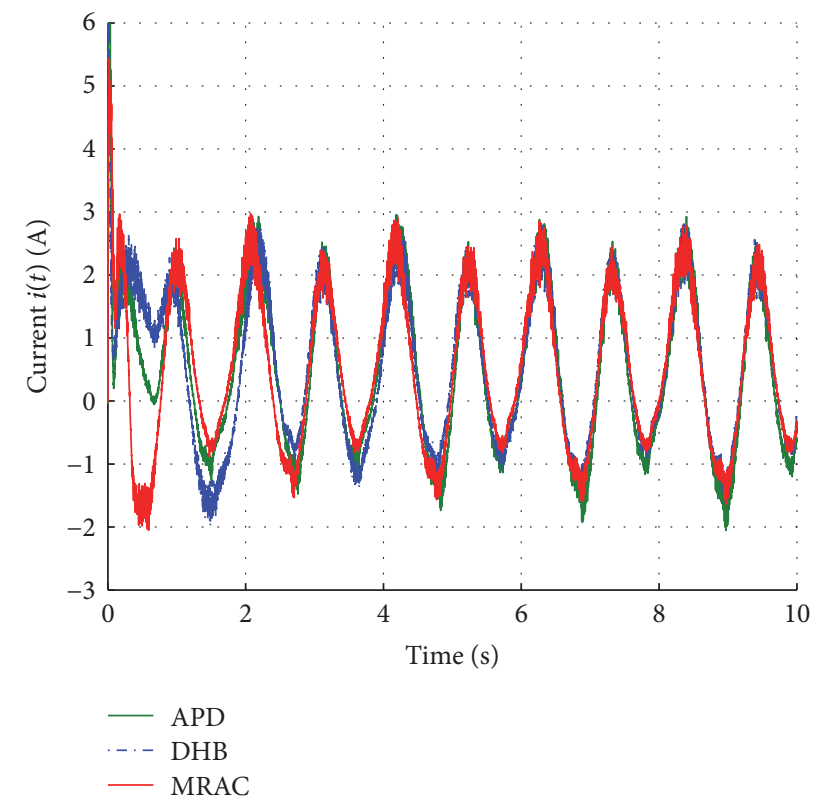

FIGURE 5: Time evolution of the electric current $i(t)$ for all the compared algorithms.

Similarly, in Figure 8, the time evolution of the estimated parameters $\widehat{L}(t), \widehat{R}(t)$, and $\widehat{K}_{B}(t)$ of the electrical part is shown. The mechanical parameters of the APD controller in (75) are not shown in Figure 7 because their values are out of scale and do not provide any meaningful information to the comparison. Likewise, the estimated values of the lumped parameters $\widehat{\boldsymbol{\theta}}_{e}(t)$ in (61) from the DHB controller are also omitted.

It is worth remembering that matrix $\mathbf{P}$ in (41) should be positive definite in order to ensure that the trajectories $\mathbf{x}(t)$

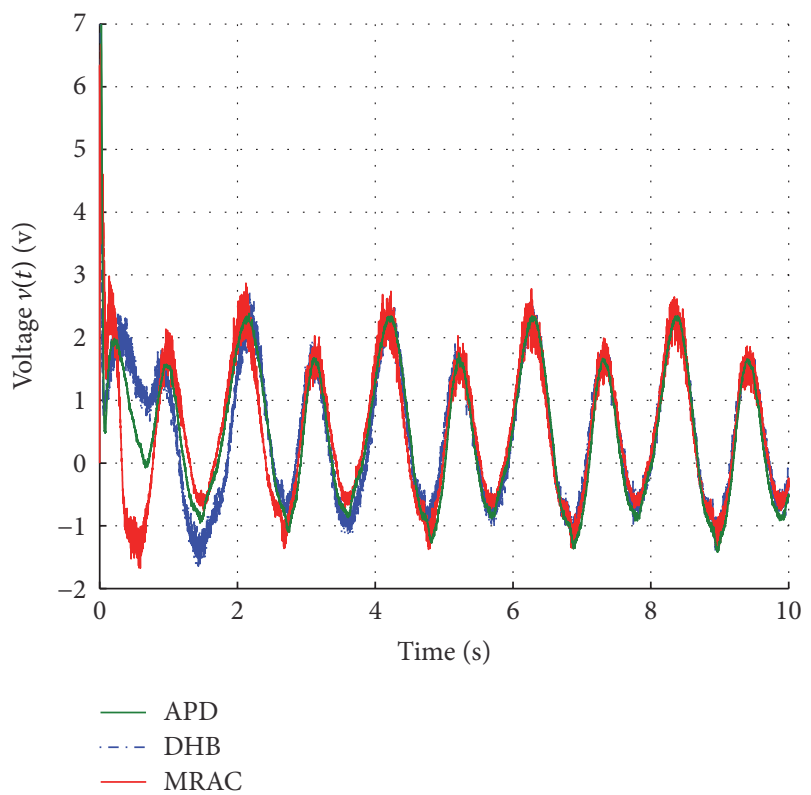

Figure 6: Time evolution of the applied voltage $v(t)$ for all the compared algorithms.
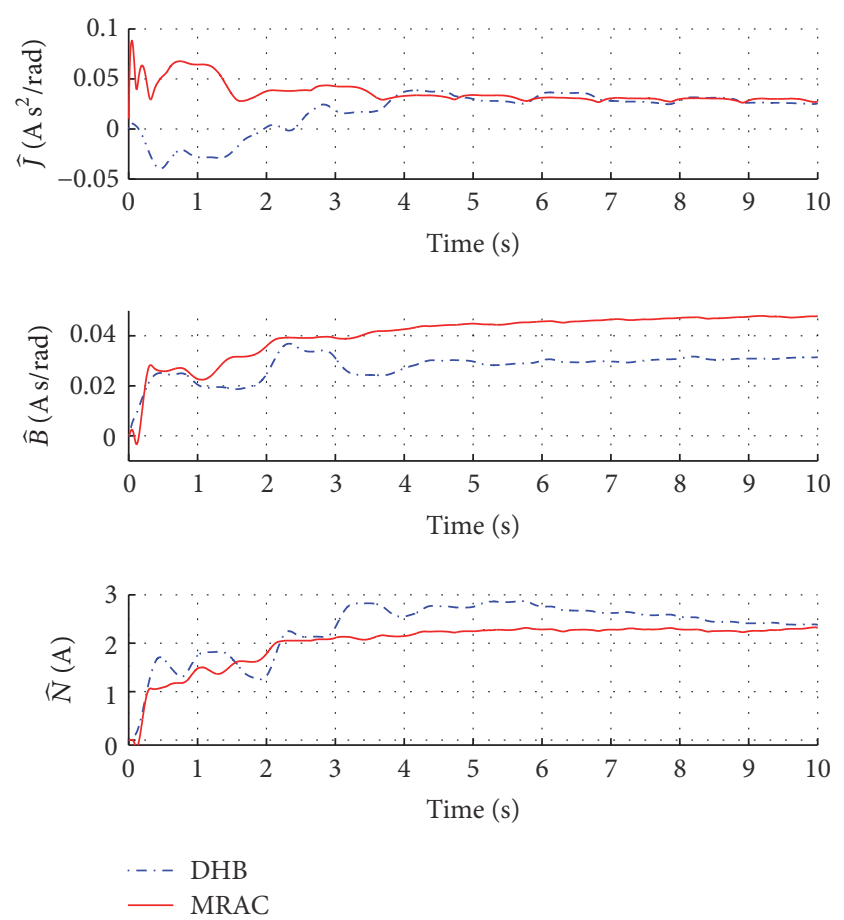

FIGURE 7: Time evolution of the estimated parameters of the mechanical part for the DHB and MRAC algorithms.

in (40) converge to zero as time $t$ approaches infinity. With the aim of demonstrating that this condition is satisfied, it is possible to use the final values of the estimated parameters in Figures 7 and 8 for $t=10[\mathrm{~s}]$, that is $\widehat{J}(10)=0.0275, \widehat{B}(10)=$ $0.0478, \widehat{R}(10)=0.8604$, and $\widehat{K}_{B}(10)=0.0364$, in addition to the control gains shown in Table 1 for the MRAC algorithm. Hence, by selecting an appropriate value for $\varepsilon$ in (41), for 

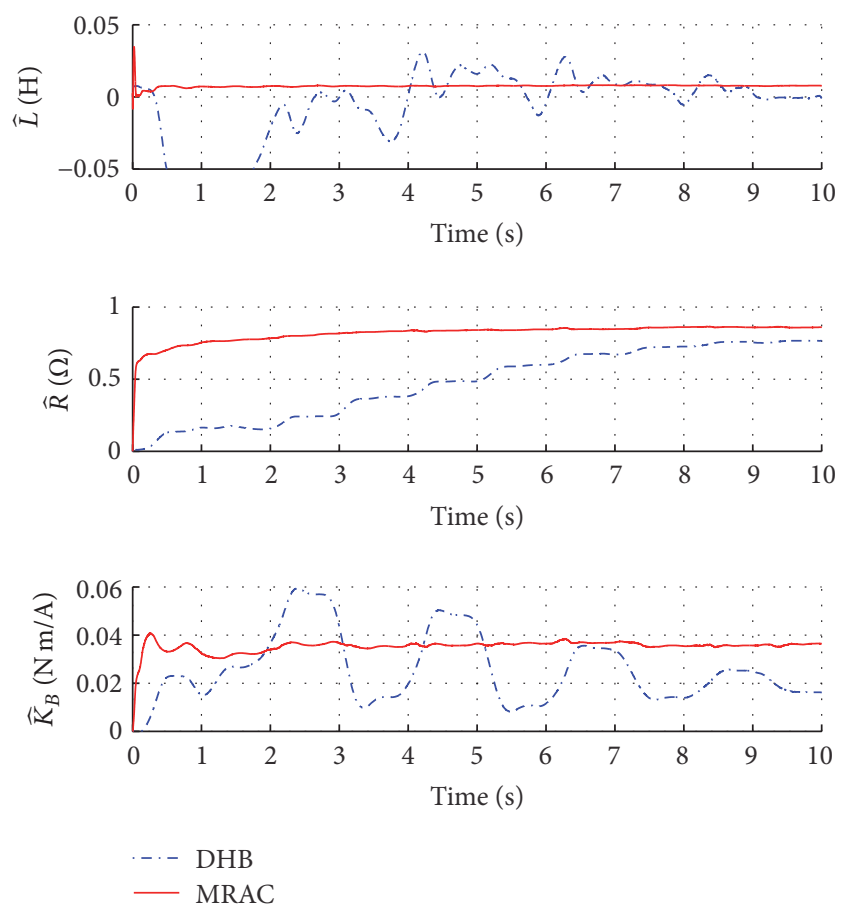

Figure 8: Time evolution of the estimated parameters of the electrical part for the DHB and MRAC algorithms.

example $\varepsilon=5$, the matrix $\mathbf{P}$ is positive definite with the eigenvalues $\lambda_{1}=9.3203, \lambda_{2}=1.0036, \lambda_{3}=0.2962$, and $\lambda_{4}=$ 0.0270 . Therefore, if rough estimates of the system parameters are available, then the provided stability analysis, specifically, the conditions to ensure the positive definiteness of matrix $\mathbf{P}$ in (41), can be used as tuning rules for the controller gains.

With the aim of complementing the results of the experimental comparison shown in Figures 4-6, Figure 9 depicts the comparison between the error signals for the three controllers, where the exponential convergence rate of the trajectory-tracking error $\widetilde{q}(t)=q_{d}(t)-q(t)$ can be seen. Furthermore, Table 4 presents a comparative of the maximum error obtained during the experiments for $15 \leq$ $t \leq 20[\mathrm{~s}]$. Specifically, we compute $\max \{|\widetilde{q}(t)|\}$ for each controller during the mentioned time interval. Table 4 also shows the maximum percentage of variation for the tracking error with respect to the total displacement of the desired trajectory $q_{d}(t)$; that is,

$$
100 \times \frac{\max \{|\widetilde{q}(t)|\}}{\max \left\{q_{d}(t)\right\}-\min \left\{q_{d}(t)\right\}} .
$$

Table 4 also shows the RMS value of the applied control voltages for each algorithm, for a time interval of $15 \leq t \leq$ $20[s]$.

The results shown in Table 4 suggest that the controller MRAC performs better than the rest of the controllers of the comparison, yielding the smallest maximum deviation with respect to the desired trajectory. The controller DHB shows the second best tracking performance on this study. The APD controller presents the worst tracking performance.

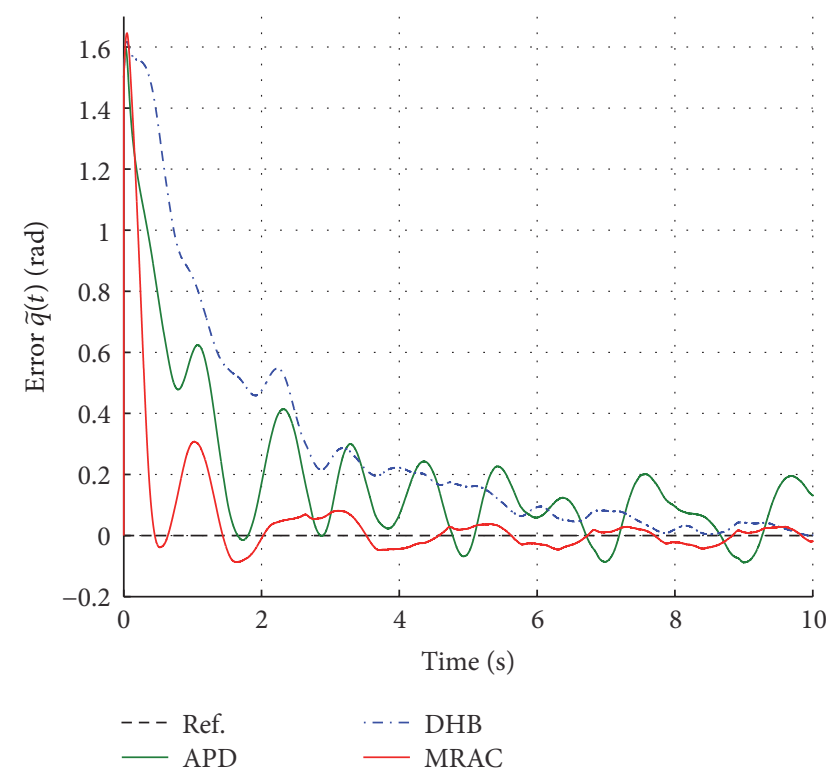

FIGURE 9: Time evolution of the trajectory-tracking error $\widetilde{q}(t)$ for all the compared algorithms.

TABLE 4: Quantification of the trajectory-tracking error $\widetilde{q}(t)$ and the applied control voltaje $v(t)$ for $15 \leq t \leq 20$.

\begin{tabular}{lccc}
\hline Index/algorithm & APD & DHB & MRAC \\
\hline $\max \{|\widetilde{q}(t)|\}[\mathrm{rad}]$ & 0.1949 & 0.0406 & 0.0402 \\
$\max \{|\tilde{q}(t)|\}[\%]^{*}$ & 4.8726 & 1.0141 & 1.0051 \\
$\operatorname{RMS}\{\tilde{q}(t)\}[\mathrm{rad}]$ & 0.1094 & 0.0266 & 0.0217 \\
$\operatorname{RMS}\{v(t)\}\left[V_{\text {rms }}\right]$ & 1.1215 & 1.1247 & 1.1267 \\
\hline
\end{tabular}

${ }^{*}$ With respect to the total displacement of the desired trajectory $q_{d}(t)$, see (86).

Note that the RMS value of the applied voltage $v(t)$ given in Table 4 is similar for the three controllers. Therefore, it is possible to say that the proposed MRAC algorithm yields better performance with practically the same applied energy.

It was also observed that the MRAC controller and the DHB scheme (controllers with armature current feedback) performed much better than the APD algorithm giving an improvement of $80 \%$ and $75 \%$, respectively. However, from the engineering point of view, the APD adaptive controller may be preferred in some cases, since is a simpler controller with respect to those that use armature current feedback. A possible advantage of the APD controller is that the applied control voltage $v(t)$ is a noise free signal (see Figure 6) as a result of not including the dirty current measurement $i(t)$.

\section{Concluding Remarks}

This paper has dealt with the trajectory-tracking control of a RLED robot with 1-DOF, for which the parameters of the dynamic model are unknown. A new controller has been proposed based on a MRAC principle. Asymptotic tracking of the desired trajectory has been proved theoretically and practically. The new controller has been compared with respect to 
an adaptive controller previously reported in literature and a controller designed by neglecting the electrical part of the system.

The new scheme showed better performance even with the adaptation of less parameters with respect to the DHB scheme, avoiding with this the overparameterization of the model. In other words, the new MRAC only requires adaptation of the six parameters present in the system, while the DHB scheme is based on adaption of nine parameters.

The results for the three controllers given in Figure 9 show that the position error signal $\widetilde{q}(t)$ is kept in a certain bound after some finite time $T>0$ (practical stability). The reasons are the quantization disturbance of position measurement, noisy current measurement, and discrete implementation, among others.

The experimental comparison indicated that, in general, better results in the tracking performance are achieved if current feedback is added to the controller. This is clearly observed from Figure 9. The reason is that the assumption of neglecting the electrical dynamics is not valid for some operating conditions. This study suggests that good performance can be obtained with feedback of position, velocity, and a "small amount" of current feedback.

Finally, as future work, the noise reduction in the control signal $v(t)$ can be investigated, which would improve the tracking performance. This can be done by using an estimation of the current signal $i(t)$ instead of using a noisy measurement of such signal, avoiding the requirement of a current sensor. In this sense, some preliminary work is presented in [41, 42], where the adaptive control of robot manipulators is considered including the actuator dynamics.

\section{Competing Interests}

The authors declare that they have no competing interests.

\section{Acknowledgments}

This work was partially supported by CONACyT, Project no. 176587, and SIP-IPN, Mexico.

\section{References}

[1] S. Zak, Systems and Control, Oxford University Press, New York, NY, USA, 2003.

[2] M. Koksal, F. Yenici, and A. N. Asya, "Position control of a permanent magnet DC motor by model reference adaptive control," in Proceedings of the IEEE International Symposium on Industrial Electronics (ISIE '07), pp. 112-117, Istanbul, Turkey, June 2007.

[3] B. Bhushan and M. Singh, "Adaptive control of DC motor using bacterial foraging algorithm," Applied Soft Computing, vol. 11, no. 8, pp. 4913-4920, 2011.

[4] S. Hwang and R. Carmichael, "Adaptive tracking control for a DC motor," in Proceedings of the 1st IEEE Regional Conference on Aerospace Control Systems, pp. 231-235, Westlake Village, Calif, USA, May 1993.

[5] R. Guenther and L. Hsu, "Variable structure adaptive cascade control of rigid-link electrically-driven robot manipulators," in Proceedings of the 32nd IEEE Conference on Decision and Control. Part 3 (of 4), pp. 2137-2142, San Antonio, Tex, USA, December 1993.

[6] D. M. Dawson, Z. Qu, and J. J. Carroll, "Tracking control of rigid-link electrically-driven robot manipulators," International Journal of Control, vol. 56, no. 5, pp. 991-1006, 1992.

[7] V. M. Hernández-Guzmán, V. Santibáñez, and G. Herrera, "Control of rigid robots equipped with brushed DC-motors as actuators," International Journal of Control, Automation and Systems, vol. 5, no. 6, pp. 718-724, 2007.

[8] J. Orrante-Sakanassi, V. Santibañez, and J. Moreno-Valenzuela, "Stability analysis of a voltage-based controller for robot manipulators," International Journal of Advanced Robotic Systems, vol. 10, article 22, 2013.

[9] R. Silva-Ortigoza, J. R. García-Sánchez, J. M. Alba-Martínez et al., "Two-stage control design of a buck converter/DC motor system without velocity measurements via a $\Sigma-\Delta$ - modulator," Mathematical Problems in Engineering, vol. 2013, Article ID 929316, 11 pages, 2013.

[10] R. Silva-Ortigoza, V. M. Hernández-Guzmán, M. AntonioCruz, and D. Muñoz-Carrillo, "DC/DC buck power converter as a smooth starter for a DC motor based on a hierarchical control," IEEE Transactions on Power Electronics, vol. 30, no. 2, pp. 1076-1084, 2015.

[11] R. Bai, "Neural network control-based adaptive design for a class of DC motor systems with the full state constraints," Neurocomputing, vol. 168, pp. 65-69, 2015.

[12] N. Wang, J. Yu, and W. Lin, "Positioning control for a linear actuator with nonlinear friction and input saturation using output-feedback control," Complexity, vol. 21, no. 2, pp. 191-200, 2016.

[13] Z. J. Chen and P. A. Cook, "Robustness of model-reference adaptive control systems with unmodelled dynamics," International Journal of Control, vol. 39, no. 1, pp. 201-214, 1984.

[14] E. Unkauf and D. Torrey, "Direct model reference control of an induction motor," in Proceedings of the IEEE 10th Annual Applied Power Electronics Conference (APEC '95), pp. 192-196, IEEE, Dallas, Tex, USA, March 1995.

[15] A. S. Kumar, M. S. Rao, and Y. S. K. Babu, "Model reference linear adaptive control of DC motor using fuzzy controller," in Proceedings of the IEEE Region 10 Conference (TENCON '08), pp. 1-5, Hyderabad, India, November 2008.

[16] H. M. Schwartz, "Model reference adaptive control for robotic manipulators without velocity measurements," International Journal of Adaptive Control and Signal Processing, vol. 8, no. 3, pp. 279-285, 1994.

[17] H. Schwartz, "An MRAC output feedback controller for robot manipulators," in Proceedings of the 7th Mediterranean Conference on Control and Automation, pp. 2292-2301, Haifa, Israel, June 1999.

[18] B. Rashidi, M. Esmaeilpour, and M. R. Homaeinezhad, "Precise angular speed control of permanent magnet DC motors in presence of high modeling uncertainties via sliding mode observerbased model reference adaptive algorithm," Mechatronics, vol. 28, pp. 79-95, 2015.

[19] C. Aguilar-Avelar and J. Moreno-Valenzuela, "Model reference adaptive control for the trajectory tracking of a DC motor with pendular load," in Proceedings of the 14th IASTED International Conference Intelligent Systems and Control, pp. 266-273, Marina del Rey, Calif, USA, November 2013. 
[20] L. Hsu, M. C. Teixeira, R. R. Costa, and E. Assunção, "Lyapunov design of multivariable MRAC via generalized passivation," Asian Journal of Control, vol. 17, no. 5, pp. 1484-1497, 2015.

[21] M. Hosseinzadeh and M. J. Yazdanpanah, "Performance enhanced model reference adaptive control through switching non-quadratic Lyapunov functions," Systems \& Control Letters, vol. 76, pp. 47-55, 2015.

[22] A. P. Nair, N. Selvaganesan, and V. R. Lalithambika, "Lyapunov based PD/PID in model reference adaptive control for satellite launch vehicle systems," Aerospace Science and Technology, vol. 51, pp. 70-77, 2016.

[23] R.-J. Wai and R. Muthusamy, "Design of fuzzy-neural-networkinherited backstepping control for robot manipulator including actuator dynamics," IEEE Transactions on Fuzzy Systems, vol. 22, no. 4, pp. 709-722, 2014.

[24] R.-J. Wai and R. Muthusamy, "Fuzzy-neural-network inherited sliding-mode control for robot manipulator including actuator dynamics," IEEE Transactions on Neural Networks and Learning Systems, vol. 24, no. 2, pp. 274-287, 2013.

[25] S. Sefriti, J. Boumhidi, R. Naoual, and Y. Boumhidi, "Adaptive neural network sliding mode control for electrically-driven robot manipulators," Control Engineering and Applied Informatics, vol. 14, no. 4, pp. 27-32, 2012.

[26] M. M. Fateh and S. Khorashadizadeh, "Robust control of electrically driven robots by adaptive fuzzy estimation of uncertainty," Nonlinear Dynamics. An International Journal of Nonlinear Dynamics and Chaos in Engineering Systems, vol. 69, no. 3, pp. 1465-1477, 2012.

[27] R.-J. Lian, "Intelligent controller for robotic motion control," IEEE Transactions on Industrial Electronics, vol. 58, no. 11, pp. 5220-5230, 2011.

[28] G. G. Rigatos, "Adaptive fuzzy control of DC motors using state and output feedback," Electric Power Systems Research, vol. 79, no. 11, pp. 1579-1592, 2009.

[29] S. N. Huang, K. K. Tan, and T. H. Lee, "Adaptive neural network algorithm for control design of rigid-link electrically driven robots," Neurocomputing, vol. 71, no. 4-6, pp. 885-894, 2008.

[30] R.-J. Wai and P.-C. Chen, "Robust neural-fuzzy-network control for robot manipulator including actuator dynamics," IEEE Transactions on Industrial Electronics, vol. 53, no. 4, pp. 13281349, 2006.

[31] J. P. Hwang and E. Kim, "Robust tracking control of an electrically driven robot: adaptive fuzzy logic approach," IEEE Transactions on Fuzzy Systems, vol. 14, no. 2, pp. 232-247, 2006.

[32] C. Kwan, F. L. Lewis, and D. M. Dawson, "Robust neuralnetwork control of rigid-link electrically driven robots," IEEE Transactions on Neural Networks, vol. 9, no. 4, pp. 581-588, 1998.

[33] C. J. Fallaha, M. Saad, H. Y. Kanaan, and K. Al-Haddad, "Sliding-mode robot control with exponential reaching law," IEEE Transactions on Industrial Electronics, vol. 58, no. 2, pp. 600-610, 2011.

[34] S. Islam and X. P. Liu, "Robust sliding mode control for robot manipulators," IEEE Transactions on Industrial Electronics, vol. 58, no. 6, pp. 2444-2453, 2011.

[35] H. Hu and P.-Y. Woo, "Fuzzy supervisory sliding-mode and neural-network control for robotic manipulators," IEEE Transactions on Industrial Electronics, vol. 53, no. 3, pp. 929-940, 2006.

[36] L. Wang, T. Chai, and L. Zhai, "Neural-network-based terminal sliding-mode control of robotic manipulators including actuator dynamics," IEEE Transactions on Industrial Electronics, vol. 56, no. 9, pp. 3296-3304, 2009.
[37] M. M. Fateh and S. Khorashadizadeh, "Optimal robust voltage control of electrically driven robot manipulators," Nonlinear Dynamics, vol. 70, no. 2, pp. 1445-1458, 2012.

[38] M. M. Fateh, H. A. Tehrani, and S. M. Karbassi, "Repetitive control of electrically driven robot manipulators," International Journal of Systems Science. Principles and Applications of Systems and Integration, vol. 44, no. 4, pp. 775-785, 2013.

[39] D. M. Dawson, J. Hu, and T. C. Burg, Nonlinear Control of Electric Machinery, Marcel Dekker, New York, NY, USA, 1998.

[40] S. Sastry and M. Bodson, Adaptive Control: Stability, Convergence and Robustness, Prentice Hall, Englewood Cliffs, NJ, USA, 1989.

[41] Y. S. Khaligh and M. Namvar, "Adaptive control of robot manipulators including actuator dynamics and without joint torque measurement," in Proceedings of the IEEE International Conference on Robotics and Automation (ICRA '10), pp. 46394644, Anchorage, Alaska, USA, May 2010.

[42] H. Kazemi and M. Namvar, "Adaptive compensation of actuator dynamics in manipulators without joint torque measurement," in Proceedings of the 52nd IEEE Conference on Decision and Control (CDC '13), pp. 2294-2299, IEEE, Firenze, Italy, December 2013. 


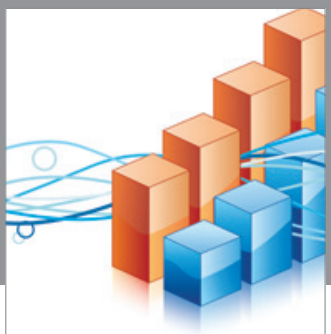

Advances in

Operations Research

vatem alat4

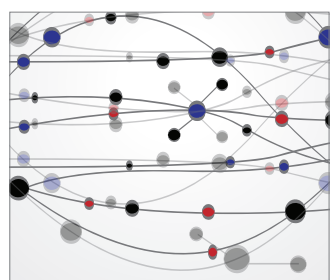

\section{The Scientific} World Journal
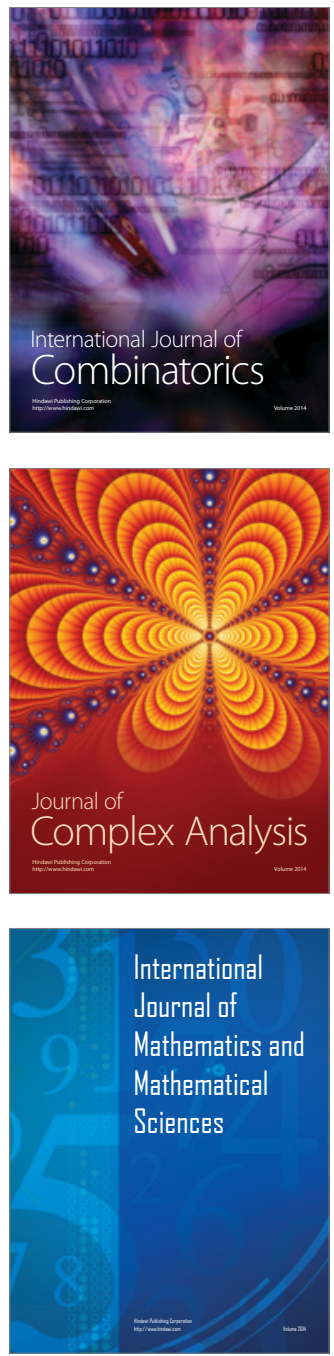
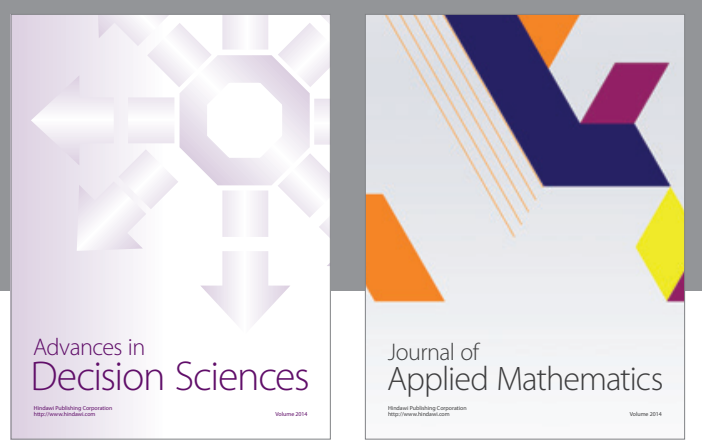

Algebra

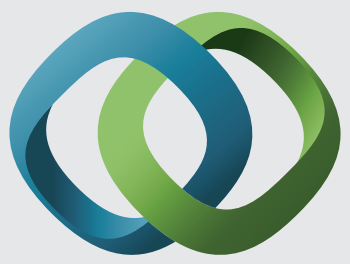

\section{Hindawi}

Submit your manuscripts at

https://www.hindawi.com
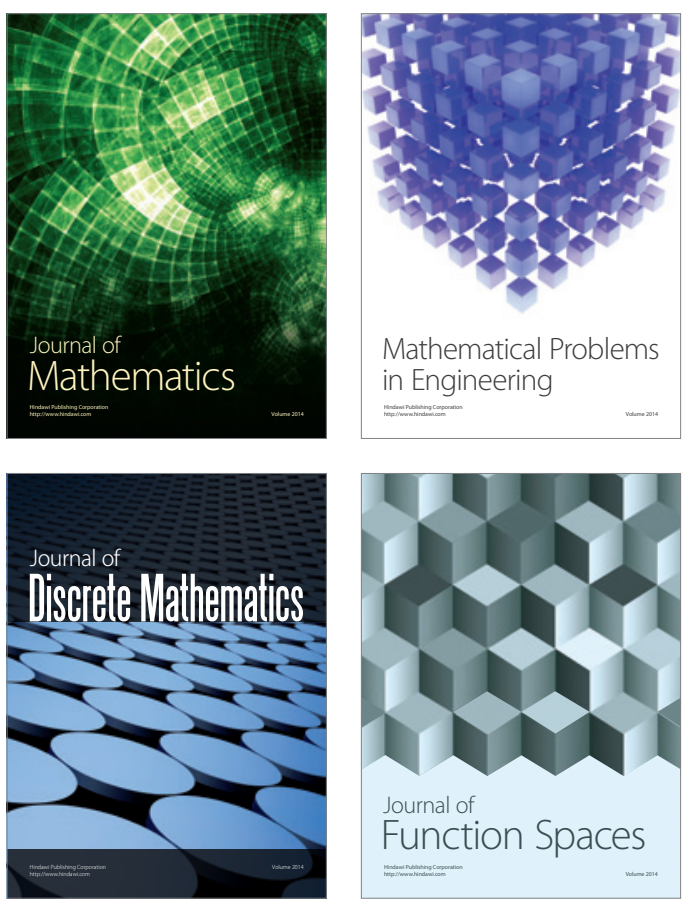

Mathematical Problems in Engineering
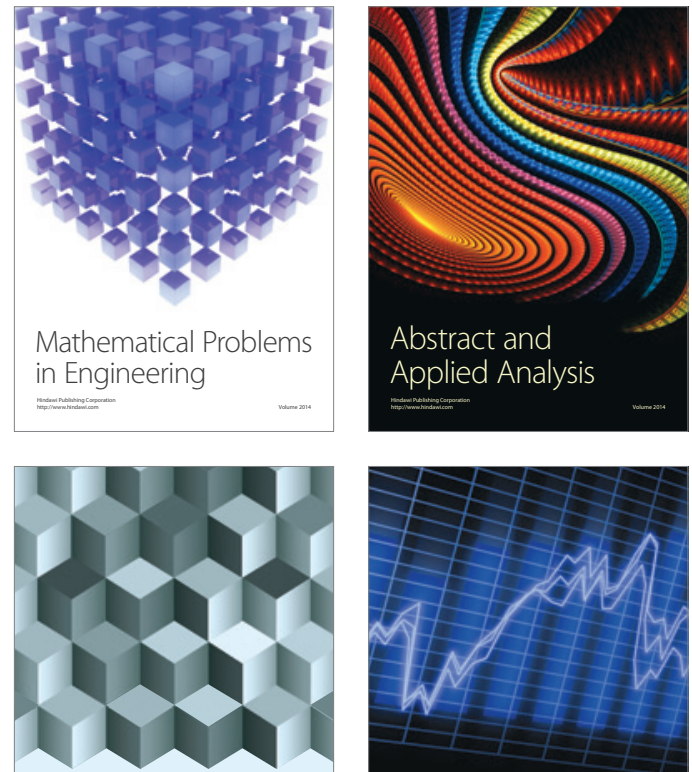

Journal of

Function Spaces

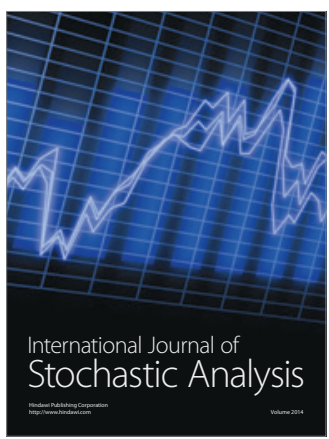

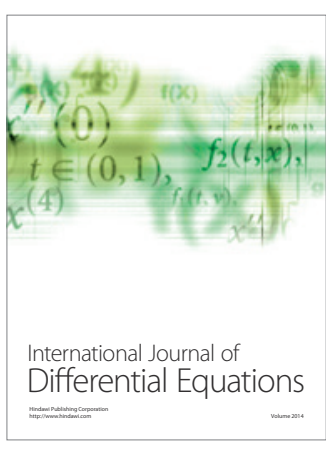
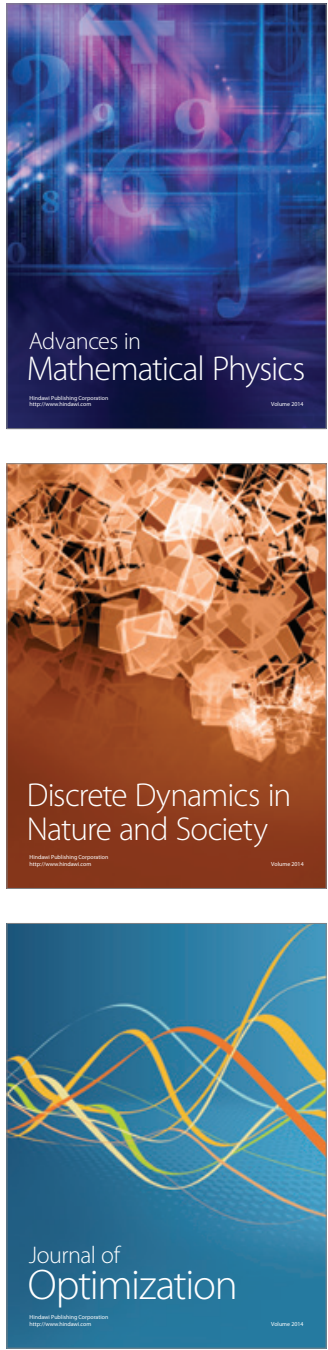